\title{
Post natal rupture of meconium pseudocyst in a neonate with delayed diagnosis
}

\begin{abstract}
Meconium peritonitis is a rare fetal disease with an increased rate of morbidity and mortality in the neonatal period. Meconium pseudocyst is a complication of meconium peritonitis which is a sterile chemical peritonitis due to intrauterine bowel perforation. When the perforation in the intestine does not heal and communication with the cyst persist postnatal that can lead to cyst expansion, infection of the cyst or rupture of pseudocyst. This is a case report of a neonate with rupture of meconium pseudocyst causing perforation peritonitis. There was a delay in diagnosis in our case, but was successfully managed with staged enterostomy.
\end{abstract}

Keywords: Ruptured meconium pseudocyst, delayed diagnosis, staged enterostomy
Volume 8 Issue 2 - 2018

\section{Basavaraju Mamatha,' Zachariah Ninan ${ }^{2}$}

'Pediatric Surgery, Department of Pediatric Surgery, Pondicherry Institute of Medical Science, India

${ }^{2}$ Pediatric Surgery Head of Department Pediatric Surgery,

Pondicherry Institute of Medical Science, India

Correspondence: Mamatha Basavaraju, C block I4 number PIMS Hospital Quarters, Ganapathychettikulum, Kalapet, Pondicherry 6050 I4,Tel 9789192634, India, Email Mamathanaveen3@gmail.com

Received: January 29, 2018 | Published: March 26, 2018

\section{Introduction}

Meconium peritonitis is defined as a sterile chemical peritonitis that is caused by the escape of meconium from the intestinal tract into the general peritoneal cavity during the fetal period. The course of the disease may range from spontaneous healing to a fatal outcome depending on the time of peroration and whether the perforation persists even after birth. Meconium peritonitis leads to substantial morbidity and mortality as high as $50-80 \%^{1}$ in the third world health setting. Early recognition in the antenatal and perinatal period, finding the underlying etiology and specific management based on the type of meconium peritonitis are key steps in providing a good outcome.

\section{Case report}

A $1.9 \mathrm{Kg}$ preterm baby was born at 33 weeks gestation by LSCS for abruption placenta. Baby developed RDS and needed intubation and ventilation with administration of surfactant. She required ventilation for 7 days followed by CPAP support. Baby developed acinetobacter sepsis which was treated with appropriate antibiotics Baby was passing meconium during the first five days and had no abdominal distension though trophic feeds were not tolerated. Mild abdominal distension was noted on eighth day which was attributed to sepsis, CPAP and RDS. Baby developed constipation, followed by increase in abdominal distension and NG aspirates. A mass was noticed below the liver on $12^{\text {th }}$ day and left lateral decubitus $\mathrm{x}$-ray film showed Pneumoperitonium (Figure 1). At laparotomy a $5 * 5 \mathrm{cms}$ thick walled cyst firmly adherent to the under surface of the liver was seen with perforation and spillage of meconium. Small intestine loops were adherent in this area with formation of a mass. After the bowel loops were separated the hepatic flexure of colon was seen entering the cyst and exiting out distally (Figure 2). Complete excision of the cyst with involved intestine was not possible due to dense adhesions to liver. Hence the cyst was opened, wall partially excised and mucosa from the involved segment of colon inside the cyst was stripped and removed (Figure 3). Ascending colon was brought out as stoma and transverse colon as mucous fistula. Post op recovery was uneventful. At 4 months baby was admitted with adhesive obstruction and treated with laparotomy and release of adhesions. At 6 months of age baby was seen thriving well and the stoma was closed after ensuring patency of distal bowel by distal colon gram. Baby is on regular follow up, feeding well and gaining weight.

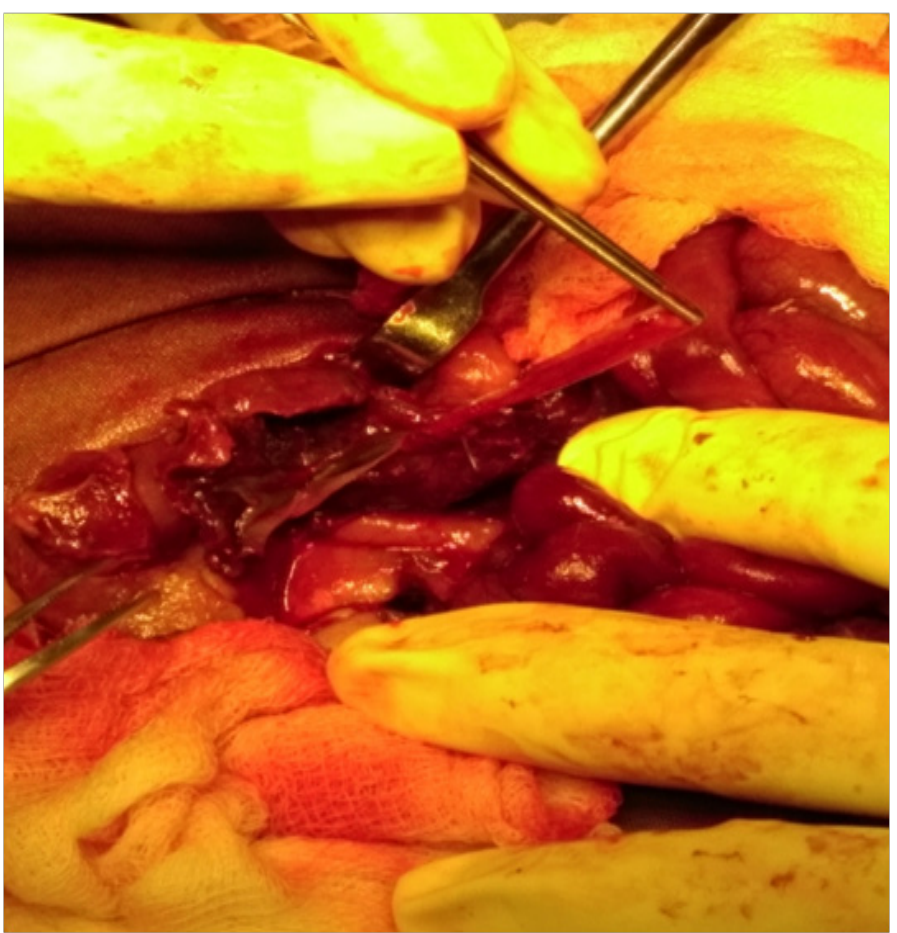

Figure I Left lateral decubitus view showing pneumoperitonium. 


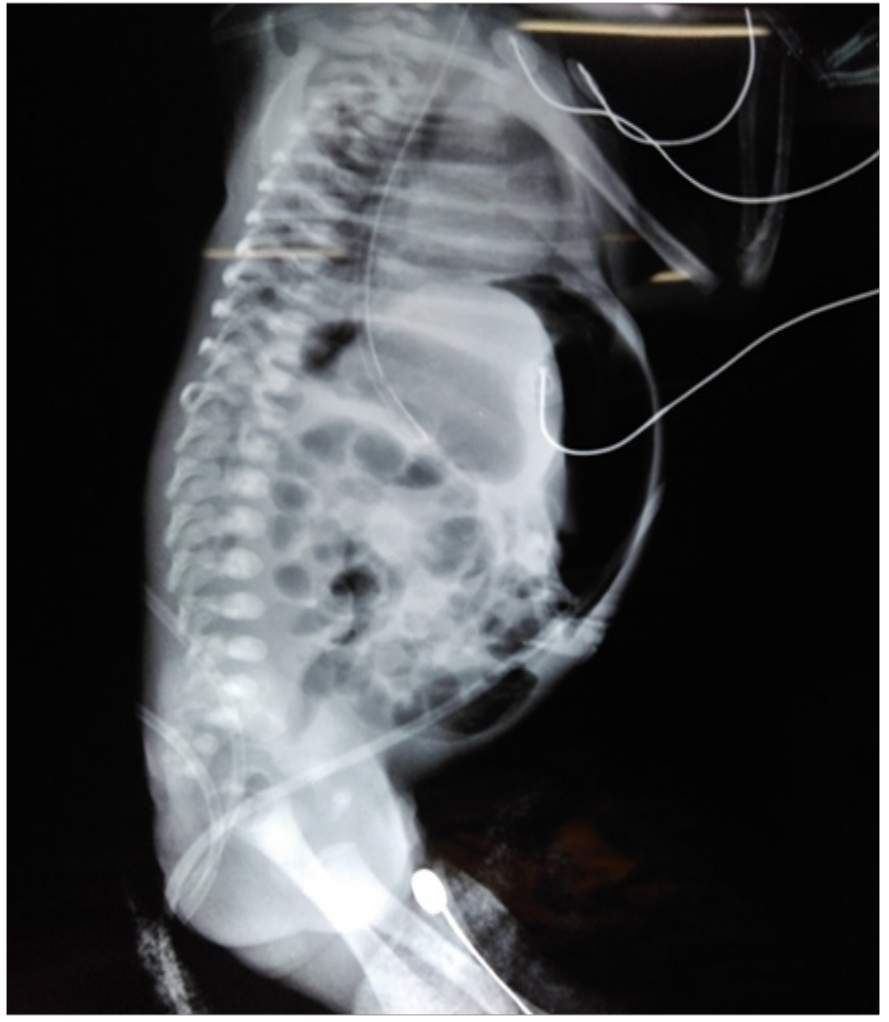

Figure 2 Perforation in the meconium cyst and hepatic flexure of transverse colon communicating with the cyst.

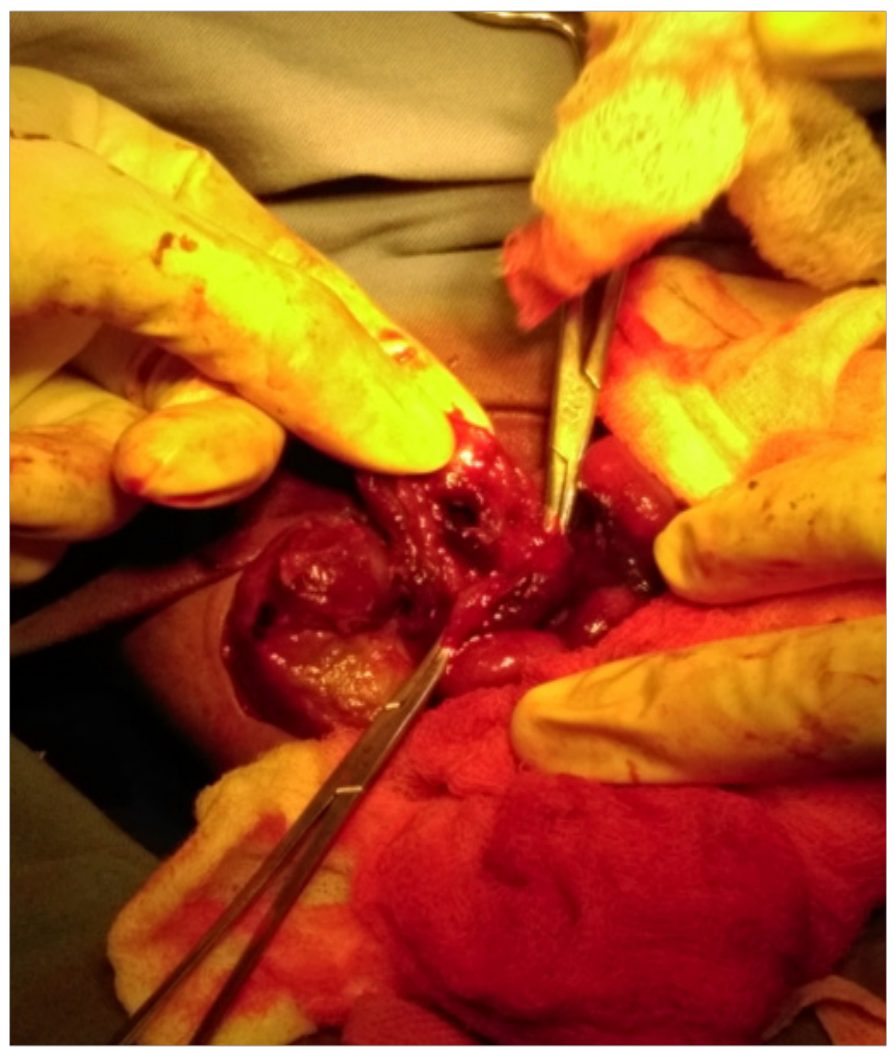

Figure 3 Mucosal stripping of the colon densely adherent to liver.

\section{Discussion}

Meconium peritonitis is defined as a sterile chemical peritonitis due to leak of meconium from intestine into peritoneal cavity during intrauterine period. The perforation causing this can occur anywhere from $4^{\text {th }}$ to $6^{\text {th }}$ week of gestation to several hours after birth. ${ }^{2}$ The incidence is 1 in 30,000 live births. ${ }^{1}$ The proposed etiology for perforation include ischemic bowel lesions associated with mechanical obstruction like atresias, volvulus, extrinsic congenital bands, meconium ileus, internal hernias, intussusceptions, meckels diverticulum, Hirschsprungs disease and idiopathic cause. ${ }^{3}$ The mortality in the past was high as $50-60 \%$ but now reduced to less than $10 \%^{1}$ and mostly due to antenatal diagnosis and improved neonatal intensive care. Meconium peritonitis pseudo-cyst (MPC) accounts for $12 \%$ of meconium peritonitis. ${ }^{4}$ In our child the antenatal scan was done at 20 weeks which was normal. Baby was delivered at 33 weeks due to abruption placenta and there were no scan in between this period to know if there was an antenatal event. When we reviewed the $\mathrm{X}$ rays taken for ARDS, $\mathrm{X}$ ray on day 1 and 2, abdomen appeared normal but on day 3 of life there were signs of free air in peritoneum which was missed. Baby was passing meconium normally for first 5 days and associated sepsis and ARDS obscured the early diagnosis for our baby. Though X ray showed features of Pneumoperitonium on day 3 the overt signs of peritonitis like bilious aspirates and abdominal distension appeared much later on day 12 . The clinical progression to peritonitis might be slow probably because, the gut continuity was maintained with no distal obstruction and use of broad spectrum antibiotic for treatment of sepsis. Our intra operative findings confirmed a ruptured meconium pseudocyst (Figure 3). We could not find any underlying etiology as there was no evidence of atresia. Meconium ileus and Hirschsprungs disease was ruled out based on clinical course and meconium history and colonic biopsy. Ileal atresia, volvulus and idiopathic are the most common causes and meconium illus is more common cause in Caucasian population.

Etiology can vary depending on the loco regional background of the study. When it is idiopathic $80 \%$ of neonates are found to be associated with neonatal anoxia or respiratory distress and hypoxic injury to splanchnic circulation is postulated. ${ }^{3}$ There are 4 types of meconium peritonitis, fibro adhesive, cystic, generalized and healed. In cystic type there is chemical peritonitis causing intestinal inflammation which become fixed around the site of perforation and form a cystic cavity. Cyst wall is fibrous and densely adherent to mesentery. If the intestinal continuity is maintained it may get sealed off and gradually heal without any intervention and the cyst size may gradually regress. ${ }^{5}$ but if there is persistent communication it may enlarge is size to form a giant cystic Meconium peritonitis or perforate. We could find only two previous case report of pseudocyst with Pneumoperitonium in literature. Saleh et al., ${ }^{4,6}$ studied antenatal diagnosed meconium peritonitis and compared patients requiring surgical intervention postnatal. They observed that most patients with pseudocyst or fetal bowel dilatation were more likely to require some surgical intervention postnatal. ${ }^{1}$ In other study by Palizzo et al. on the cystic type of MP they suggested there may be a potentially rapid lethal course especially due to persistence of leak or re rupture of the cyst with new meconium spillage into abdomen and hence some form of surgical intervention always indicated at birth. ${ }^{7}$ There are some studies which state that meconium pseudocyst is different from cystic meconium peritonitis and there is very little literature on the same. ${ }^{8,9}$ According to a review by Masashi minato et al. ${ }^{8}$ the pseudocyst 
consists of dilated thinned out intestine filled with meconium that has smooth muscle layer connecting the cyst to normal intestine but epithelium lost due to inflammation.

In contrast, the cystic-type meconium peritonitis has a fibrous wall. But many case reports use the term pseudocyst to any meconium peritonitis -cystic type. ${ }^{1}$ our case was unusual because, the cyst had ruptured to cause a peritonitis which occurs rarely, the pneumo peritoneum was missed and diagnosis delayed. But on exploration the contamination was limited to the right side of abdomen and the intestinal continuity maintained without any distal obstruction which might have contributed to the good outcome. The cyst was dissected from the surrounding adhesion and the entrapped perforated intestine resected and healthy bowel exteriorized. Many reports of conservative drainage of cyst at first followed by a primary anstomosis have been reported with good outcome ${ }^{10}$ after confirming distal patency. But we followed a conventional approach of initial diversion and later anastomosis because of contamination and delayed diagnosis. Irregular antenatal scans, missed Pneumoperitonium on $\mathrm{X}$ ray, slow clinical progression of the disease (due to maintained intestinal continuity and antibiotic use) may have contributed to the delay in diagnosis. Associated co morbidity can cause a delay in presentation and delay in diagnosis of meconium peritonitis especially if it is not antenatal diagnosed. Meconium pseudocyst if ruptures like our case can be successfully treated with staged enterostomy.

\section{Conclusion}

Meconium pseudocyst can present in postnatal period with rupture and peritonitis. Sometimes the diagnosis can be delayed and missed especially if not diagnosed antenatal. Meconium pseudocyst which ruptures to form a generalized peritonitis can be successfully treated with staged enterostomy.

\section{Acknowledgment}

None.

\section{Conflict of interest}

None.

\section{References}

1. Saleh N, Geipel A, Gembruch U, et al. Prenatal diagnosis and postnatal management of meconium peritonitis. J Perinat Med. 2009;37(5):535538.

2. Packard GB, Reynolds LE. Meconium peritonitis. Ann Surg. 1951;133(4):548-554.

3. Jose Boix Ochoa, J Lloret. Meconium peritonitis. In: Prem Puri, editor. Newborn surgery, $2^{\text {nd }}$ ed. Arnold publishers; 2003;pp. 471-472.

4. Khalil Al Tawil, Walid Salhi, Safiah Sultan, et al. Does Meconium Peritonitis Pseudo-Cyst Obstruct Labour? Case Reports in Obstetrics and Gynecology. 2012:3.

5. Basu S, Kumar A, Pandey N, An unusual cause of meconium peritonitis in a foetus. Journal of Paediatrics and Child Health, 2009;45(4):231-233.

6. Wong A, Toh C, Lien R, et al. Prenatal MR imaging of meconium pseudocyst extending to the right subphrenic space with right lung compression. Pediatr Radiol. 2006;36(11):1208-1211.

7. Pelizzo G, Codrich D, Zennaro F, et al. Prenatal detection of the cytic form of meconium peritonitis: no issue for delayed postnatal surgery. Pediatr Surg Int. 2008;24(9):1061-1065.

8. Minato M, Okada T, Miyagi H, et al. Meconium pseudocyst with particular pathologic findings: a case report and review of the literature. $J$ Pediatr Surg. 2012;47(4):e9-e12.

9. Park HG, Kim KS, Choi SY, et al. Diagnostic Usefulness of CT in Meconium Pseudocyst in a Newborn: A Case Report. J Korean Soc Radiol. 2014;70(2):159-162.

10. Nam SH, Kim SC, Kim DY, et al. Experience with meconium peritonitis. $J$ Pediatr Surg. 2007;42(11):1822-1825. 